Research Article

\title{
Systematic Study on Expression of Vocal Music and Science of Human Body Noise Based on Wireless Sensor Node
}

\author{
Yifei Xu (iD \\ Yulin University School of Art, Yulin 719000, Shaanxi, China \\ Correspondence should be addressed to Yifei Xu; xuyifei@yulinu.edu.cn \\ Received 11 March 2021; Revised 7 April 2021; Accepted 21 April 2021; Published 29 April 2021 \\ Academic Editor: Mian Ahmad Jan \\ Copyright (C) 2021 Yifei Xu. This is an open access article distributed under the Creative Commons Attribution License, which \\ permits unrestricted use, distribution, and reproduction in any medium, provided the original work is properly cited. \\ In this paper, we use a wireless sensor node to investigate the relationship between vocal art composition and human body noise. \\ For the vocal music, the suggested predictor created a wireless sensor node convergence link model and the HART graph routing \\ model is used to systematically design the human noise science. The wireless sensor node optimizes the acquisition of information \\ on vocal art and analyzes the acoustic signal characteristics. The optimally balanced scheduling model has been used to sci- \\ entifically and distributedly regulate vocal art performance and human body noise. The proposed model enhances the signal \\ detection ability and raises the performance level of vocal art, music art, and human body noise signal. The results of the \\ simulation demonstrate that the proposed model enhances the signal acquisition capability of vocal music and the analysis of \\ human noise. It has a powerful ability to generate vocal signal characteristics and a stronger ability to handle interference \\ with noise.
}

\section{Introduction}

The vocalist's performance, like all musical works, is dependent on the singer or performer's ability to reproduce the composer's work. The goal of singing is to use powerful vocal abilities to effectively convey the ideological content of songs, to infect the audience with haunting melodies, and to make the audience enjoy the art [1]. The human body is a marvelous human organ for the singer; its interpretation, expression, representation, and embodiment of human musical art is unmatched in any musical instrument in the world. Master the correct breathing technique as well as the correct voice. The sound's high position relates to complete headspace resonance, which we stress as mask resonance [2]. A focused, bright, relaxed, and penetrating sound resonates with the entire cavity of the head. Singing is more relaxing, and it sounds brighter and more wonderful [3]. Humming practice should help you achieve a high position sound. It seems to be that human body noise and transcription have a systemic connection with vocal music art expression, and it is useful to research the relationship model between vocal music art expression and the science of body noise in a person [4]. The Vocal Art Performance Network is suggested in conjunction with wireless sensors to capture artistic information of vocal music and gain control over the improvement of human body noise [5]. Network channel equation design and optimization of path detection algorithms, as well as path conflict avoidance design, are the fundamental technologies of vocal art expression and systemic control of human body noise science [6].

Wireless sensors are scientific and systemic ways of monitoring the expression of sound and noise art in the human body [7]. The optimization of the network causation node was governed through the design of routing protocol optimization and control of the wireless sensor route of the Internet of Things. Some research findings were discovered, including a network activation node that suggested a scientific and systemic control method for the human voice of wireless sensors without sound and performance [8]. To increase human body noise position, adaptive modulation coding has been used. Maximum sensor power management is used, and as a result, network node coverage capacity is increased [9]. This procedure, however, has issues with low stability and consistency in gathering voice art information 
from wireless sensors and systemic monitoring of human body science. A scientific and systematic method of human noise control and data collection based on wireless sensors with a strong and appealing understanding of node communication load is proposed in [10]. Wireless sensor nodes constructed the topological model of the routing graph of the voice information distribution route, checked the combined load and power of the wireless Internet sensor of things, and performed network routing conflict prevention design. There are other problems with the control mechanism, such as less interference and less autonomous deployment capability [11]. It also investigates noise settings, noise extraction facilities, sound sampling quality, storage format, age of recorded items, number of recorded items, vocal music, and other topics and is of high standard and seriousness [12]. The study meets the standards by distinguishing between men and women, but the sound portion of the recorded object is unclear, and the kinds of sound signal collection and singing range are not defined. The preceding content is one of the most important factors determining the performance of Sound Music Art [13].

This research presents a mathematical framework for vocal expression and the science of human noise based on wireless sensors and a wireless node of convergence sensor model of vocal art. The HART graph routing model is used to create a structured design for vocal art and noise science. The wireless sensor is designed to make the best use of node models, and the convergent connection gain control technique is used to control wireless sensor vocal art performance. Furthermore, the adaptive balanced scheduling model is used for scientific and distributed tracking of vocal art performance as well as human body noise. The network of wireless sensors for acquiring information about vocal art is intended to better acquire information on vocal art and understand sound signal characteristics. We also enhance our ability to voice and detect the signal. Finally, simulation experiments are conducted in order to demonstrate the best performance of this technique in scientific and systemic performance and human noise analysis.

\section{Methodology}

In this section, we introduce vocal data using wireless sensors in order to control human corporal noise on a scientific and systematic level. Additionally, we develop an artistic expression of vocal music and a distribution model of human noise control nodes in wireless sensor and vocal performance, as well as a convergence link model for human noise control nodes.

2.1. Voice Signal Preprocessing. The extraction of acoustic parameters is critical to the objective assessment of the body voice. The voice signal must be processed in advance, including digital, preaccentuation, framing, and window addition, regardless of the procedure used to extract and evaluate the parameters $[14,15]$. Supplementation is an important step in analyzing voice signals and extracting acoustic parameters. People's speech signals are analog, so the analog signals must be converted to digital signals before the digital signals can be processed and analyzed [16]. In this paper, a valuable and digitized voice data file is obtained by recording the singing voice signal with a microphone and a computer sound card and collecting it for later use [17]. After receiving the data file of the human voice signal, the signal is reweighted, and frame and window preprocessing is performed, which allows the signal after processing to satisfy the real needs; it is critical to increasing the accuracy of the subsequent data processing. Figure 1 depicts a noise signal that has been preconditioned [18].

2.2. Frame and Window. The generation of speech signals is related to various movements of the organs of speech, and the random movement of the organs causes the signal itself to be unstable. However, the state of vocal organ changes is much slower than that of sound vibration [19]. Therefore, it is considered that the speech signal is stable within $10-30 \mathrm{~ms}$, and there is no obvious change in the characteristics of the speech spectrum and some physical properties in the short term. Therefore, only the speech signal is divided into a few short speech segments, the processing method of the stationary signal can be introduced into the speech signal processing, and each segment here is called a short speech analysis frame. Each frame signal is regarded as a stationary process, which overcomes the difficulty of the speech signal analysis and processing, and the processing of each frame is equal to the long signal with invariant features. $\mathrm{N}$ sampling points are combined into one observation unit, called frame length. Usually, the frame length is 256 or 512, and the time is about $20-30 \mathrm{~ms}$.

The framing should be continuous; that is, it is not possible to replicate the frame, and the information is not presented and not processed between frames. The solution to this issue is to overlap the components so that frame and structure can maintain a smooth transition, which includes samples $M$, called frameshifts, usually $0-1 / 2$ frame lengths. The signal is altered after framing from the original signals as the frames at start and end are stopped and need a window to be attached. Adding windows to the signal can not only effectively solve the problem of the discontinuity of the position signal at the beginning of the frame, but also make the original nonperiodic speech signal present the part of the periodic function. In the process of signal windowing, window functions are usually selected according to the nature and processing requirements of the signal being analyzed. The main part of the rectangular window is more concentrated, but the side lobe is high and there is a side lobe, which will transform the high-frequency interference and the leakage into the transform. It is suitable for the precise readout of the main lobe frequency but does not consider the signal processing of the amplitude accuracy. The peak value of the main lobe and the first side lobe peak decay to $40 \mathrm{~dB}$, which is suitable for the analysis of the narrowband signals with strong interference noise as shown in Figure 2 for the preprocessed noise signal.

The voice demands the involvement and collaboration of power bodies, vibrational organisms, resonance organisms, 


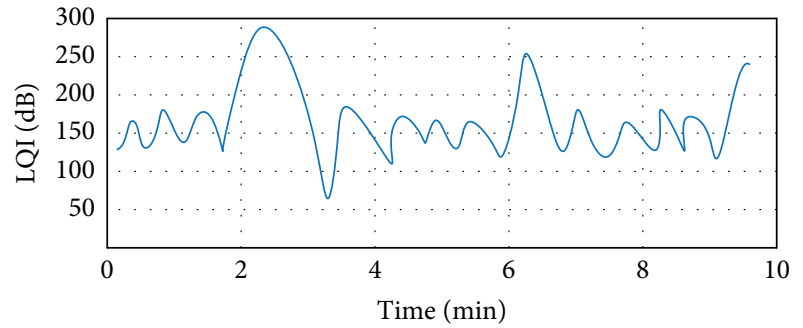

Figure 1: Preconditioning noise signal [18].

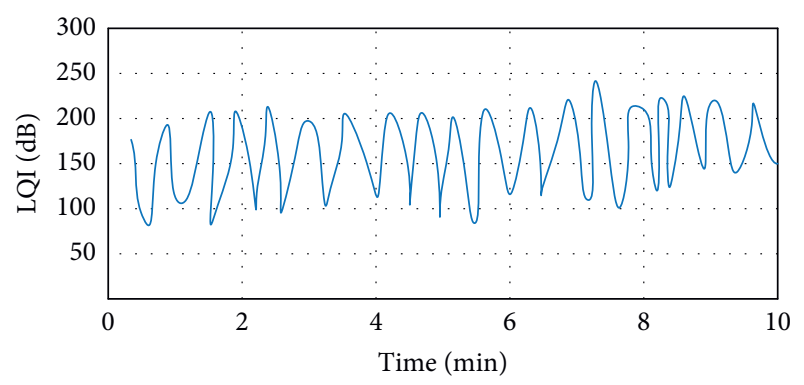

FIgURe 2: Preprocessed noise signal [19].

and the nervous system. Due to the incredibly intricate process, the voice function is a multidimensional and multiparameter evaluation of your voice with a complete and impartial assessment of your creative voice. It is important to have multidimensional acoustic signal parameters: selection and proper extraction of the technical signals of the voice utilizing vocal analysis, observation of the person's state in a pronunciation, assessment of whether the voice is normal and its regularity, and opening the doctor's diagnosis for an unbiased evaluation of the subsequent technique [20].

\subsection{Vocal Performance and Human Noise Control Nodes} Convergence Link Model. To realize the information collection of vocal art based on wireless sensors and the scientific and systematic control of human body noise, firstly, the convergence link model of vocal art expression and human noise control node of a wireless sensor is constructed. The wireless sensor vocal art monitoring area is a square area with a side length of $M$, and there are $\mathrm{N}$ vocal art sensor routing relay nodes, assuming that the base station has sufficient energy. In the process of scientific and systematic control of vocal art and human noise, the energy balance control is carried out through the adaptive allocation of laser sensor routing nodes [21]. The information acquisition node of wireless sensor vocal music art is defined asi, based on the number of neighbor nodes, the number of hops and sampling interval $T_{f}$ between source and cluster head node is determined. The power consumed by cluster head node to transmit $L$ bit data is set as $T_{s}=N_{f} T_{f}$, and the equilibrium game between cluster head node and transmission link is carried out by using the game analysis method of node transmit power. The energy consumption of each node is obtained as follows:

$$
T_{c}=\operatorname{ent}\left(T_{f} / N_{c}\right) .
$$

The distributed routing detection algorithm is used to analyze the coverage of nodes through the shortest path deployment. Whenc ${ }_{j} T_{c}<T_{f} \forall j \in\left[0, N_{f}-1\right]$, the base station of the wireless sensor network with vocal performance and human noise is located at the top of the monitoring area of the network at the initial moment [8]. The data packet forwarding of wireless sensor dominating point set is $\sum_{j \in S, j \neq i} x_{j} r_{j}(x) p_{j}^{i}(x)$. The characteristics of output power spectral density of vocal performance and human noise wireless sensor network converging link are obtained by using the conflict coordination control method:

$$
p_{i}=\frac{\sigma^{2}}{1-\sum_{j=k+1}^{n} \gamma_{i} / \gamma_{j}+G} \frac{\gamma_{i}}{h_{i}\left(\gamma_{i}+G\right)},
$$

where $\gamma_{i}=\gamma_{t h}$, and the sensor coefficient representing the artistic performance of the wireless sensor and the energy saving distribution of the nodes of the human noise control node estimates the minimum variance of the wireless sensor node $\eta$ at any position and obtains the triggered node. The objective function of energy balance control is described as follows:

$$
\arg \max _{p_{i} \in P_{i}} u_{i}=\arg \max _{p_{i} \in P_{i}} \gamma_{i}=\frac{\gamma_{t h} \sigma^{2}}{h_{i}\left[G-(n-k-1) \gamma_{t h}\right]},
$$

where $\operatorname{argmax}_{p_{i} \in P_{i}} u_{i}$ is the frame head length of cluster head node $i$. The maximum likelihood estimation results of the convergence link model for the stability control of the vocal art performance sensor can be expressed as follows:

$$
\sigma_{\eta}^{2}=E[\hat{x}(\eta)-x(\eta)]^{2}, \quad \eta \in \Omega_{\eta},
$$

where $\hat{x}(\eta)$ is the coverage of the sensor network based on the equivalent model of the vocal performance of the wireless sensor and the convergence link gain control of the human noise control node is constructed, and the trigger control between the vocal art performance and the human noise control node is carried out [22].

\subsection{HART Graph Routing Algorithm and Routing Detection} Protocol Design. The HART graph routing model is used to design the route detection protocol for vocal performance and human noise wireless sensor networks. The HART map routing detection protocol is constructed based on the IEEE 802.3 EFM protocol, and $M_{1} M_{2} \cdots M_{N}$ are the human body noise distribution under the wireless sensor. In the optimal network topology, the network transmission channel $K\left\{r_{i}\right\}$ is a two-way channel wireless transceiver channel, which is represented $\operatorname{as} r_{i}(x)$, where $m u(i, x)+n p$ is the network node distribution density of the noise coverage area of the human body, and the network transmission channel $m u(i, \mathbf{x})+n p>0$ is the wireless transceiver channel of a twoway channel [9]. According to the HART graph routing model, the difference characteristic function of node output is obtained: 


$$
E s(j)=\{(u, v) \in E \mid u \in V s(j), v \in V s(j)\} .
$$

The trigger node of the vocal art expression and the human body noise wireless sensor network is transformed into the link holomorphic analytical model, and the trigger node is repaired to obtain the relationship between the vocal music art expression and the human body noise wireless sensor network. The $2 \mathrm{D}$ coordinate system of the routing node is represented by $\left(x_{u}, y_{u}\right)$, and the artistic representation of vocal music is equivalent to the gain control model of the convergence link of the human noise control node in the following form:

$$
\max _{p_{i} \in P_{i}} u_{i}\left(p_{i}, p_{-i}\right)=\ln \left(1+\gamma_{i}\right)-\beta_{i} \gamma_{i}, \quad i \in N .
$$

Based on the two-way link model $(k, i)$, the vocal art expression of wireless sensor, and the design of human noise acquisition routing detection protocol model, the accuracy percentage of cluster head emission data is obtained:

$$
\eta_{\text {comm }}=\frac{k_{1} \cdot l}{E_{\text {comm }}} \cdot\left(1-p_{\text {drop }}\right) \text {. }
$$

The cluster head node transmit data packet is $\mathrm{CH}_{i}\left(i \in \mathrm{C}_{1}\right)$, the channel equalization control method is used to calculate the average communication time interval of the first node [10]. For a given link gain coefficient $\gamma_{t h}$, when $\beta_{c_{1}}=u_{i}^{\prime}\left(\gamma_{t h}\right)$, vocal performance and human noise wireless sensor network, the overlay gain transfer function of the complex is described as

$$
\frac{\partial u_{i}}{\partial p_{i}}=\frac{G h_{i}}{\sum_{j \neq i} h_{j} p_{j}+\sigma^{2}}\left(\frac{1}{1+\gamma_{i}}-\frac{1}{1+\gamma_{t h}}\right) .
$$

The average amount of mutual information for the $j$ reference node is

$$
\begin{array}{r}
R_{i}(k)=\operatorname{diag}\left\{r_{(i-1) q+1}(k), r_{(i-1) q+2}(k), \ldots, r_{(i-1) q+q}(k)\right\} \\
(i=1,2, \ldots, N) .
\end{array}
$$

From this, the correlation matching function between the artistic expression of vocal music and the root node and trigger node of the human body noise wireless sensor network is described as follows:

$$
\begin{aligned}
& \breve{B}(k)=E\left[w(k) \breve{V}^{T}(k)\right], \\
& =E\left[w(k) V^{T}(k)\right]\left[L^{-1}(k)\right]^{T}, \\
& =\left[\begin{array}{lllll}
\breve{B}_{1}(k) & \breve{B}_{2}(k) & \cdots & \breve{B}_{N}(k)
\end{array}\right] \text {, }
\end{aligned}
$$

where

$$
\left\{\begin{array}{l}
\Phi(k)=[A(k)-J(k) \breve{H}(k)], \\
\breve{w}(k)=\Gamma(k) w(k)-J(k) \breve{V}(k) .
\end{array}\right.
$$

According to the above processing, a routing detection protocol based on the HART model and wireless network sensors is established.

\section{Control Optimization Realization}

In this section, we will discuss the collection of vocal music art information, as well as the performance of vocal music art and the realization of scientific systemic control optimization for human noise. In addition, we will also analyze and control the convergence link between vocal performance and the human noise control node.

3.1. Analysis and Gain Control of Convergence Link. Vocal music is conducted by using a vocal art format convergence model and the wireless sensor network human noise control node, as well as a routing detection protocol between vocal art expression and the wireless body noise sensor network. It optimizes the art of vocal music and the research and systematic management of human noise. The human noise science systematic control approach is used to control the wireless sensor's vocal art performance, as well as the convergent link control for the node of human noise control and the path between the vocal art expression and the human noise wireless sensors.

The numbers of conflicts and the delay are $\alpha_{l}$ and $\tau_{l}$, respectively, where $l \in[0, L-1] \tau_{0}<\tau_{1}<\ldots \tau_{L-1}$, and the robust sexy radius $\operatorname{GNInter}_{i}(n)$ of trigger node can be expressed as

$$
\operatorname{NInter}_{i}(n)=\operatorname{NInter}_{i}(n)+1, \quad \text { if } j \notin N_{i} \cup t_{i j} \geq T_{h} .
$$

If $\operatorname{CIntra}_{i}(n)$ is used to represent the energy overhead of the transmission of F-bit packets by node $i$ and the maximum threshold for transmission packets of node CInter ${ }_{i}(n)$, then the allocated power and time chips for each cluster head node are

$$
\begin{aligned}
& \operatorname{CIntra}_{i}(n)=\frac{\operatorname{NIntra}_{i}(n)}{T}, \\
& \operatorname{CInter}_{i}(n)=\frac{\operatorname{NInter}_{i}(n)}{T} .
\end{aligned}
$$

Based on this, the routing conflict avoidance mechanism of the Internet of things is designed, and the maximum probability density function of sensor node $i$ effectively covering the monitoring area $U_{i}(n)$ in thet $(n)$ time window is obtained as follows:

$$
U_{i}(n)=\alpha \operatorname{CIntra}_{i}(n)+(1-\alpha) \operatorname{CInter}_{i}(n), \quad \alpha \in(0,1) .
$$

By optimizing the topology design of the routing diagram of the vocal art expression and the human body noise wireless sensor network, the robustness coefficient of the vocal art expression and the human body noise wireless sensor network routing graph topology is obtained.

$$
E_{\mathrm{Rx}}(l)=l E_{\text {elec }} .
$$

Among them, $d$ is the artistic representation of vocal music and the routing transfer probability of the 
transmission node of the human noise wireless sensor network is the residual energy $r$ of the cluster head, which is the current communication cycle of vocal art expression and human body noise wireless sensor network. Therefore, the convergent link gains control of vocal performance, and the human noise control node is realized. The optimized control objective function is as follows:

$$
T(n)=\left\{\begin{array}{l}
\frac{P}{1-P[r \bmod (1 / P)]} \cdot\left\{c\left[\frac{E_{\text {res }}}{E_{\text {init }}}+\left(r_{u} d i v \frac{1}{p}\right)\left(1-\frac{E_{\text {res }}}{E_{\text {init }}}\right)\right]+(1-c) \frac{d_{\max }-d_{i}}{d_{\max }-d_{\text {min }}}\right\} n \in G, \\
0,
\end{array}\right.
$$

where when vocal art performance and human body noise wireless sensor network trigger node election as cluster head, set $\mathrm{ru}$ is 0 ; when there is a routing conflict, set the sensor transmission node connectivity cost coefficient equal to threshold $\mathrm{d} 0$; the residual energy of the triggered node gradually decreases; and vocal performance and human noise control node convergence link gain control matrix $\mathrm{BN} \times 1$ are as follows:

$$
\begin{aligned}
r(t) & =\sum_{i} \sum_{j=0}^{N_{f}-1} \sum_{l=0}^{L-1} b_{i} \alpha_{l} p\left(t-i T_{s}-j T_{f}-c_{j} T_{c}-\tau_{l}\right)+\omega(t), \\
& =\sum_{i} \sum_{j=0}^{N_{f}-1} b_{i} p_{h}\left(t-i T_{s}-j T_{f}-c_{j} T_{c}-\tau_{0}\right)+\omega(t) .
\end{aligned}
$$

Multiplying the vocal performance with the node link matrix $\mathrm{SN} \times L$ of the human body noise wireless sensor network and the energy matrix TL $\times 1$ consumed by the node to receive the $l$ bits data, thus realizing the expression of the vocal music art of the wireless sensor and the human body. Route detection and link gain control of noise control nodes.

3.2. Cluster Head Transmit Power Equalization Control and Adaptive Equalization Scheduling. A control model equalizes voice performance and human noise with cluster head power. Wireless sensor networks are installed to implement the wireless sensor; the node is used for the optimal wireless sensor deployment and as an approach to the convergent link gain control. A balanced adaptive planning model is used to regulate vocal art and the noise of the human body scientifically. The communication load between wireless sensor vocal art performance and human noise control node is as follows:

$$
\begin{aligned}
r(t) & =\sum_{i} \sum_{j=0}^{N_{f}-1} \sum_{l=0}^{L-1} b_{i} \alpha_{l} p\left(t-i T_{s}-j T_{f}-c_{j} T_{c}-\tau_{l}\right)+\omega(t), \\
& =\sum_{i} \sum_{j=0}^{N_{f}-1} b_{i} p_{h}\left(t-i T_{s}-j T_{f}-c_{j} T_{c}-\tau_{0}\right)+\omega(t),
\end{aligned}
$$

$$
p_{h}(t)=\sum_{l=0}^{L-1} \alpha_{l} p\left(t-\tau_{l, 0}\right) .
$$

In addition, $\omega(t)$ is the weight of routing conflict avoidance for intracluster nodes and $p_{h}(t)$ is the power attenuation of $l$ bits data sent by wireless sensor vocal art information acquisition nodes. The payload overhead of the routing topology for the vocal performance and human noise wireless sensor network cluster heads and trigger nodes is obtained as follows:

$$
P_{\mathrm{AOMDV}}=\left(1-P_{d}\right)^{2}\left\{1-\left[1-\left(1-P_{e}\right)^{n}\left(1-P_{d}\right)^{n-1}\right]^{m}\right\} \text {. }
$$

The routing conflict avoidance mechanism of the Internet of things is constructed and the RMS error of node robustness control is obtained by using the maximum likelihood estimation model. The calculation formula is expressed as follows:

$$
\begin{aligned}
\mathrm{MSE} & =\xi=E\left[\varepsilon_{k}^{2}\right], \\
& =E\left[d_{k}^{2}\right]-2 W^{T} P+W^{T} R W .
\end{aligned}
$$

The message coverage of the triggering node has been strengthened because of the performance of the vocal music, the load of the human noise wireless sensor network, and the robustness perception coefficient from the source to the sink. The game model for robustness control of the information acquisition node of the wireless sensor sound music art is achieved as follows, following an autoadaptable rotation schedule:

$$
\begin{aligned}
E_{T x}(L, d) & = \begin{cases}L E_{\text {elect }}+L \varepsilon_{f s} d^{2}, & d<d_{0}, \\
L E_{\text {elect }}+L \varepsilon_{m p} d^{4}, & d<d_{0},\end{cases} \\
E_{R x}(L) & =L E_{\text {elect }}
\end{aligned}
$$

\section{Analysis of Simulation Experiment}

In this section, we examine and evaluate the performance and effectiveness of the proposed model and test the application apprehending wireless sensor's network for the vocal music art and also acquisition node optimization control and the human body noise scientific systematic

where 
analysis. The experiment analysis is carried out; the experiment is carried out with MATLAB 7 simulation tool.

4.1. Experimental Setup. We have set up a wireless sensing network cluster with default configurations. The $\mathrm{C} / \mathrm{C}++$ network is used to build the related graphical model of vocal art expression and body noise. The message link model and control of the wireless sensing vocal music node are also built on the same configuration. The initial location of wireless sensor network transmission is set at 200 to 200 , the radius of communication coverage in a single node is $2 \mathrm{~m}$, the bit sequence of vocal data is $24 \mathrm{Mbit}$, and network topology occurs. There are 6 activate nodes and 12 sink nodes, with a link output delay of $2080 \mathrm{~s}$, and an estimated completion possibility is set to $\mathrm{Pe}=0.98$ with false alarm chance, that is, $\mathrm{Pd}=0.04$. The node transmission data simulation time is $100 \mathrm{~s}$ with the SNR of $3 \mathrm{~dB}$, and the acquisition results of vocal and human noise signals are shown in Figure 3.

The signal model of Figure 3 is taken as the research object, the artistic expression of vocal music and the detection of human noise signal are carried out, and the detection results are shown in Figure 4.

4.2. Detection of Human Noise Signal. To increase the control robustness of vocal music information acquisition nodes, the cluster head transmit power equalization control technique is used to implement adaptive equalization scheduling of wireless sensor nodes. The wireless sensor vocal art information collection vocal art performance and the human body noise scientific systemic control performance test simulation analysis result is shown in Table 1.

According to the analytical results in Table 1, the proposed method increases the signal collection of vocal music and the system analysis ability of the human body's noise signal. It has a robust ability to extract sound signal characteristics, a stronger ability to withstand noise interference, and a high test detection probability. Figure 5 demonstrates a performance test comparison that illustrates how this procedure can increase the accuracy of vocal and human noise detection.

In addition, Table 2 shows the medians and standard variations of diverse acquisition procedures. From Table 2, the current study's findings show that various types of data measurement techniques achieve comparable results for the chosen number of elements. In f0, F1, and F3 measures, as well as the associated statistical significance and variance factors, acquisition methods were observable. Furthermore, there were no significant changes in some time measures, such as the number of pauses and percentage pauses. Remarkably, there was some frequency and strength variability in the recording devices that remained constant.

4.3. Subjective Auditory Perception Evaluation. Individual auditory evaluation is affected by personal measures and there are issues with precision and stability. It is undeniable that subjective auditory perception assessment is the only

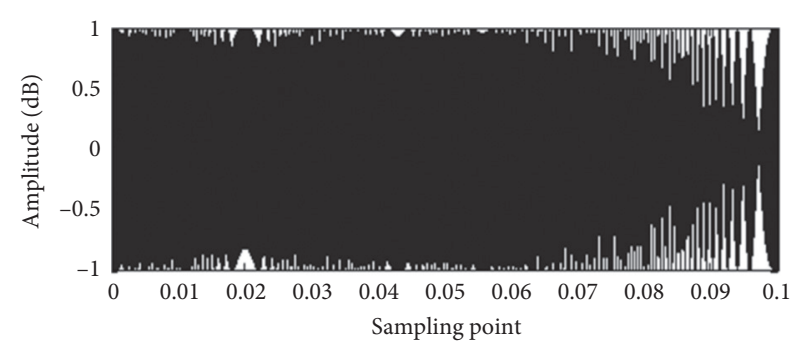

Figure 3: Acquisition results of vocal and human noise signals.

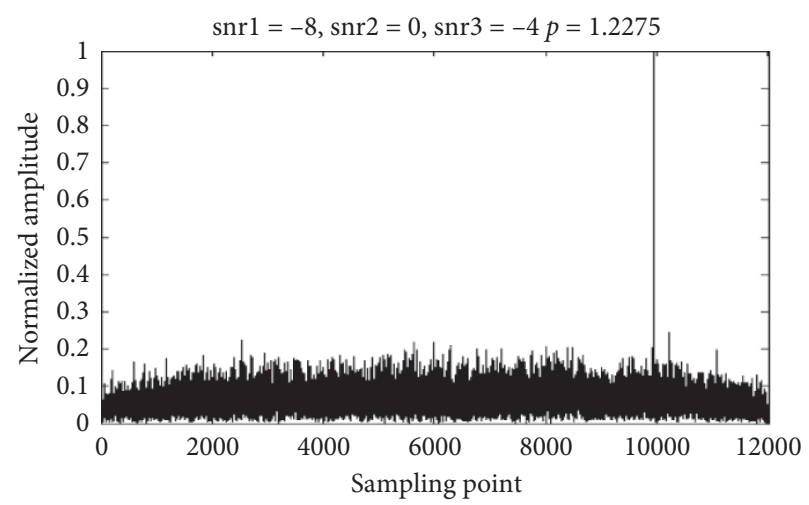

FIGURE 4: Detection result of vocal art signal based on the wireless sensor.

TABLE 1: Comparison of performance testing.

\begin{tabular}{lccc}
\hline SNR $(\mathrm{dB})$ & Proposed method & Reference [4] & Reference [6] \\
\hline-10 & 89.94 & 82.34 & 83.98 \\
-5 & 94.35 & 89.65 & 85.33 \\
0 & 98.43 & 93.21 & 92.94 \\
5 & 99.54 & 94.39 & 95.4 \\
10 & 95.63 & 96.89 & 97.83 \\
\hline
\end{tabular}

standard for testing and evaluating the performance of vocal music art as well as the validity of objective test parameters of human noise and sound function to provide directed signals for voice input training samples. The subjective auditory perception assessment of vocal voice and human noise is carried out to validate the efficacy of the human noise detection procedure. We invited two vocal music teachers and vocal professionals to listen to 41 sound recording materials individually to examine the audible perception. The score was scored by ten points and was higher than 8.5 , based on the quality, the volume, the color, and the range of the sound signals. The results were excellent with a 7.5-8.5 split. There is a mild distinction between 6.5 and 7.5 and less than 6.5. In the XLS (F0), the first resonance peak (F1, and third resonance peak (F3) is sampled for the experiment and the subjective assessment results are stored. The acoustic parameters and subjective evaluation results of each art voice signal are shown in Table 3. The subjective appraisal findings are $7,19,5$, and 10, respectively, among the 41 samples of the Art Vocals Signal. In Table 3 are shown the acoustic 


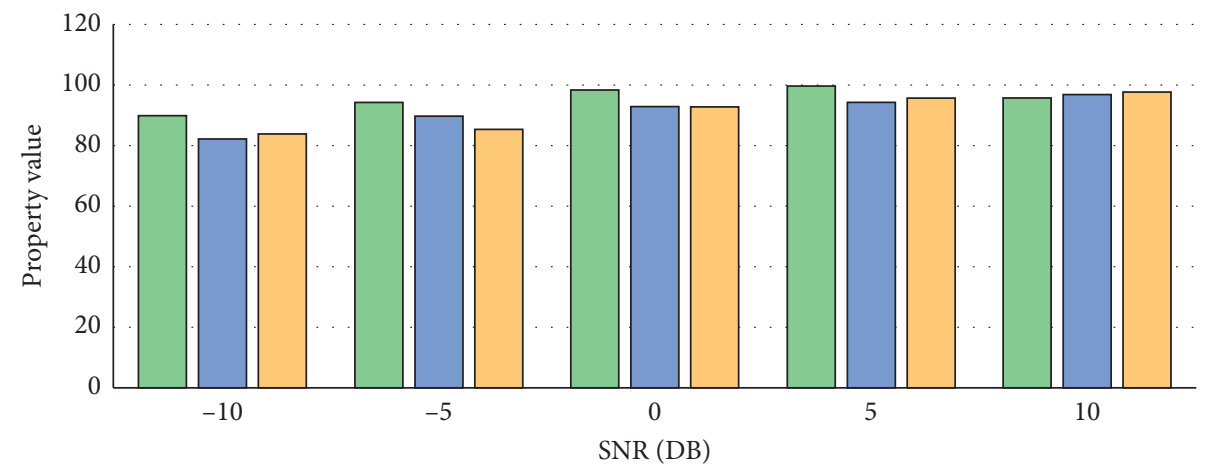

Proposed method

Reference 4

Reference 6

Figure 5: The performance test comparison.

TABLE 2: Medians and standard variations of diverse acquisition procedures.

\begin{tabular}{lccccc}
\hline \multirow{2}{*}{ Measure } & \multicolumn{2}{c}{ PC } & \multicolumn{2}{c}{ SSR } & HDD \\
& Medians & SD & Medians & SD & Medians \\
\hline Number of pauses & 35.6 & 15.04 & 43.97 & 17.18 & 42.33 \\
Pauses (\%) & 0.22 & 0.1 & 0.21 & 0.08 & 0.32 \\
F3-HZ & $1,361.34$ & 113.88 & $1,315.03$ & 117.01 & $1,321.75$ \\
F1-HZ & 752.78 & 175.21 & 695.82 & 173.38 & 721.38 \\
F0-HZ & 141.85 & 40.38 & 139.43 & 38.32 & 112.03 \\
Medians of pause length in sec & 0.31 & 0.08 & 0.27 & 0.07 & 141.31 \\
\hline
\end{tabular}

TABLE 3: Subjective auditory perception evaluation.

\begin{tabular}{|c|c|c|c|c|c|}
\hline Number & Sex & F1 (HZ) & F3 (HZ) & F0 (HZ) & Subjective evaluation results \\
\hline 1 & M & 578.5 & 2147.6 & 516.2008 & Poor \\
\hline 2 & M & 621 & 2440.8 & 518.3619 & Poor \\
\hline 3 & M & 548.8 & 2109 & 496.7788 & Poor \\
\hline 4 & M & 562.5 & 2145.6 & 506.2008 & Poor \\
\hline 5 & M & 611 & 2430.8 & 515.3679 & Poor \\
\hline 6 & M & 544.8 & 2139 & 486.7788 & Poor \\
\hline 7 & $\mathrm{M}$ & 453.1 & 2547.7 & 388.8746 & Good \\
\hline 8 & $\mathrm{M}$ & 531 & 2543.3 & 483.3791 & Good \\
\hline 9 & M & 576.9 & 2592.1 & 517.8078 & Good \\
\hline 10 & $\mathrm{M}$ & 527.8 & 2914.2 & 476.5466 & Good \\
\hline 11 & M & 568.6 & 2547.2 & 474.2042 & Good \\
\hline 12 & $\mathrm{M}$ & 433.1 & 2647.7 & 384.8746 & Good \\
\hline 13 & $\mathrm{M}$ & 521 & 2546.3 & 473.3791 & Good \\
\hline 14 & M & 575 & 2692.1 & 527.8078 & Good \\
\hline 15 & M & 527.9 & 2814.2 & 466.5466 & Good \\
\hline 16 & $\mathrm{M}$ & 558.6 & 2546.2 & 472.2042 & Good \\
\hline 17 & M & 558.6 & 2546.2 & 472.2042 & Good \\
\hline 18 & M & 400.8 & 2909 & 358.2 & Excellent \\
\hline 19 & M & 408.8 & 2845.4 & 308.256 & Excellent \\
\hline 20 & $\mathrm{M}$ & 410.8 & 2809 & 350.2 & Excellent \\
\hline 21 & $\mathrm{M}$ & 691 & 3289 & 514.1546 & Secondary \\
\hline 22 & $\mathrm{M}$ & 358.5 & 2308 & 246.155 & Secondary \\
\hline 23 & FM & 288.5 & 2138.8 & 260.6958 & Poor \\
\hline 24 & FM & 364.9 & 2152.6 & 250.7011 & Poor \\
\hline 25 & FM & 261.5 & 2135.8 & 270.6958 & Poor \\
\hline 26 & FM & 354.9 & 2172.6 & 250.7011 & Poor \\
\hline 27 & FM & 297.8 & 2454.6 & 258.8196 & Good \\
\hline 28 & FM & 335.4 & 2592.3 & 130.0283 & Good \\
\hline 29 & FM & 291.2 & 2418.2 & 226.6648 & Good \\
\hline 30 & FM & 386.2 & 2585.7 & 258.7824 & Good \\
\hline
\end{tabular}


TABLE 3: Continued.

\begin{tabular}{llcccc}
\hline Number & Sex & F1 (HZ) & F3 (HZ) & F0 (HZ) & Subjective evaluation results \\
\hline 31 & FM & 392.5 & 2998.9 & 164.2512 & Good \\
32 & FM & 296.5 & 2453.6 & 255.8196 & Good \\
33 & FM & 338.4 & 2598.3 & 168.0283 & Good \\
34 & FM & 287.2 & 2518.2 & 225.6648 & Good \\
35 & FM & 402.8 & 2885.4 & 308.9557 & Excellent \\
\hline
\end{tabular}

parameters and the subjective results of the evaluation for each voice signal.

\section{Conclusion}

This paper provides an acquisition methods framework for systematic analysis of vocal art and human body noise performance based on wireless sensors and constructs the convergence of vocal music node wireless sensor models. The HART graph routing model is used to design the vocal art and human noise sciences continuously, to create a nodeoptimal deployment model for the wireless sensor, and to control the performance of the wireless sensor's vocal art using a converging link gain control approach. The adaptive equilibrated scheduling model is used to regulate vocal performance and human body noise scientifically and distributedly. The network of wireless sensors for acquiring information about vocal art is intended to better acquire information on vocal art and understand sound signal characteristics and improve the ability to speak and detect signals. The findings of the simulation show that the method proposed must improve the ability to acquire the vocal musical signal and to analyze human noise. The teachings and instructions of vocal music performers have a high application value.

\section{Data Availability}

The datasets used and/or analyzed during the current study are available from the corresponding author on reasonable request.

\section{Conflicts of Interest}

The author declares that he has no conflicts of interest.

\section{References}

[1] Y. Pan, Z. Yan, W. Guo et al., "Pulse laser injection seeded state detector and experimental research," Laser Technology, vol. 40, no. 2, pp. 153-156, 2016.

[2] X. Lu, "Anti-interference control algorithm for UAV based on attitude fusion filter," Transducer and Microsystem Technologies, vol. 35, no. 7, pp. 116-119, 2016.

[3] C. Sun, C. Yang, S. Fan et al., "Energy efficient distributed clustering consensus filtering algorithm for wireless sensor networks," Information and Control, vol. 44, no. 3, pp. 379384, 2015.

[4] S. Chao-xuan, P. Wang, H. Zhuang-zhi et al., "Feature-level fusion recognition algorithm based on analogy decision tree classification," Control and Decision, vol. 31, no. 06, pp. 1009-1014, 2016.
[5] I. A. Kareem and M. G. Duaimi, "Improved accuracy for decision tree algorithm based on unsupervised discretization," International Journal of Computer Science and Mobile Computing, vol. 3, no. 6, pp. 176-183, 2014.

[6] A. Hu, R. Zhang, and D. Yin, "Image quality assessment using a SVD-based structural projection," Signal Processing: Image Communication, vol. 29, no. 3, pp. 293-302, 2014.

[7] L. C. Manikandan and D. R. K. Selvakumar, "A new survey on block matching algorithms in video coding," International Journal of Engineering Research, vol. 3, no. 2, pp. 121-125, 2014.

[8] Y. Zhan, H. Dai, and S. Wang, "Image quality assessment scheme with topographic independent components analysis for sparse feature extraction," Electronics Letters, vol. 50, no. 7, pp. 509-510, 2014.

[9] Z. Li, M. Song, G. Yin, X. Ma, and H. Song, "Cross deployment networking and systematic performance analysis of underwater wireless sensor networks," Sensors, vol. 17, no. 7, p. 1619, 2017.

[10] H. Zhong, K. Ma, and Y. Zhou, "Modified BM3D algorithm for image denoising using nonlocal centralization prior," Signal Processing, vol. 106, pp. 342-347, 2015.

[11] Z. C. Qin, Z. Zhou, and X. C. Zhao, "Energy efficient beamforming scheme for wireless sensor networks," Journal on Communications, vol. 34, no. 4, pp. 19-27, 2013.

[12] W. Wang, R. Wang, H. MEHRPOUYAN, and G. Zhang, "Beamforming for simultaneous wireless information and power transfer in two-way relay channels," IEEE Access, vol. 5, no. 5, pp. 9235-9250, 2017.

[13] C. Du, X. Chen, and L. Lei, "Energy-efficient optimisation for secrecy wireless information and power transfer in massive MIMO relaying systems," IET Communications, vol. 11, no. 1, pp. 10-16, 2017.

[14] J. R. A. Wang, L. Sboui, and Z. Rezki, "Power minimization of a wireless sensor node under different rate constraints," IEEE Transactions on Signal Processing, vol. 64, no. 13, pp. 34583469, 2016.

[15] R. J. Alouini, J. Kim, Z. Xie et al., "A skin-attachable, stretchable integrated system based on liquid GaInSn for wireless human motion monitoring with multi-site sensing capabilities," Npg Asia Materials, vol. 9, no. 10, p. e443, 2017.

[16] L. Sun, P. Ren, and Q. Du, "Fountain-coding aided strategy for secure cooperative transmission in industrial wireless sensor networks," IEEE Transactions on Industrial Informatics, vol. 12, no. 1, pp. 291-300, 2016.

[17] A. Wang, H. Meng, Y. F. A. Gaus et al., "Artificial intelligent system for automatic depression level analysis through visual and vocal expressions," IEEE Transactions on Cognitive \& Developmental Systems, no. 99, p. 1, 2017.

[18] A. S. Cohen, T. L. Renshaw, and K. R. Mitchell, "A psychometric investigation of "macroscopic" speech measures for clinical and psychological science," Behavior Research Methods, vol. 48, no. 2, pp. 475-486, 2016.

[19] F. Kim, S. Marguerat, and V. Shahrezaei, "Division rate, cell size and proteome allocation: impact on gene expression noise 
and implications for the dynamics of genetic circuits," Royal Society Open Science, vol. 5, no. 3, p. 172234, 2018.

[20] H. Bdi and L. J. WilliamS, "Principal component analysis," Wiley Interdisciplinary Reviews: Computational Statistics, vol. 2, no. 4, pp. 433-459, 2010.

[21] Y. Zhao and R. Zhang, "Full-duplex wireless-powered relay with self-energy recycling," IEEE Wireless Communications Letters, vol. 4, no. 2, pp. 201-204, 2015.

[22] H. Dai, Y. Huang, C. Li, and L. Yang, "Energy-efficient resource allocation for device-to-device communication with WPT," IET Communications, vol. 11, no. 3, pp. 326-334, 2017. 